\title{
Barley Stripe Rust Resistance QTL: Development and Validation of SNP Markers for Resistance to Puccinia striiformis f. sp. hordei
}

\author{
K. Esvelt Klos, T. Gordon, P. Bregitzer, P. Hayes, X. M. Chen, I. A. del Blanco, S. Fisk, and J. M. Bonman
}

First, second, third, and eighth authors: Agricultural Research Service, United States Department of Agriculture (USDA), Aberdeen, ID 83210; fourth and seventh authors: Department of Crop and Soil Science, Oregon State University, Corvallis 97331; fifth author: USDA Agricultural Research Service, Pullman, WA 99164; and sixth author: Department of Plant Sciences, University of California, Davis 95616.

Accepted for publication 17 May 2016.

\begin{abstract}
Esvelt Klos, K., Gordon, T., Bregitzer, P., Hayes, P., Chen, X. M., del Blanco, I. A., Fisk, S., and Bonman, J. M. 2016. Barley stripe rust resistance QTL: Development and validation of SNP markers for resistance to Puccinia striiformis f. sp. hordei. Phytopathology 106:1344-1351.

Quantitative trait loci (QTL) for barley stripe rust resistance were mapped in recombinant inbred lines (RIL) from a 'Lenetah' $x$ 'Grannelose Zweizeilige' (GZ) cross. GZ is known for a major seedling resistance QTL on chromosome $4 \mathrm{H}$ but linked markers suitable for marker-assisted selection have not been developed. This study identified the 4H QTL (log of the likelihood $[\mathrm{LOD}]=15.94$ at 97.19 centimorgans $[\mathrm{cM}]$ ), and additional QTL on chromosomes $4 \mathrm{H}$ and $6 \mathrm{H}(\mathrm{LOD}=5.39$ at $72.7 \mathrm{cM}$ and 4.24 at

$34.46 \mathrm{cM}$, respectively). A QTL on chromosome $7 \mathrm{H}(\mathrm{LOD}=2.04$ at $81.07 \mathrm{cM}$ ) was suggested. All resistance alleles were derived from GZ. Evaluations of adult plant response in Corvallis, OR in 2013 and 2015 provided evidence of QTL at the same positions. However, the minor QTL on $4 \mathrm{H}$ was not statistically significant in either location/year, while the 7H QTL was significant in both. The single-nucleotide polymorphism markers flanking the resistance QTL were validated in RIL from a '95SR316A' $\times$ GZ cross for their ability to predict seedling resistance. In 95SR316A $\times$ GZ, 91 to $92 \%$ of RIL with GZ alleles at the major 4H QTL and at least one other were resistant to moderate in reaction. In these populations, at least two QTL were required to transfer the barley stripe rust resistance from GZ.
\end{abstract}

Barley stripe rust, caused by Puccinia striiformis f. sp. hordei Erikss., is a threat to barley production in regions with cool, wet climates, and is capable of causing seed quality reductions and yield losses exceeding 70\% (Line 2002; Marshall and Sutton 1995). Stripe rust was commonly observed on wheat, barley, and wild grasses in western North America throughout the 20th century but impact on barley production was light, likely because the endemic pathogen was $P$. striiformis f. sp. tritici. $P$. striiformis f. sp. hordei was introduced from Europe to South America in 1975 (Dubin and Stubbs 1986), and caused significant yield losses in barley (Line 2002). In 1987, yield losses to barley of up to $50 \%$ were observed in Mexico (Brown et al. 2001; Navarro and Zamora 1990). Serious epidemics in the western United States were observed by 1995 (Brown et al. 2001; Chen et al. 1995; Line 2002; Marshall and Sutton 1995). The periodic yield losses due to stripe rust observed since have been relatively low (under 7\%), in part because of the nonrace-specific adult plant resistance present in popular barley cultivars (X. M. Chen, personal communication). Continuing efforts to incorporate resistance genes into cultivars under development will help maintain this protection against major stripe rust epidemics in barley.

Although $P$. striiformis $\mathrm{f}$. sp. hordei isolates collected in the United States encompass considerable variation (Line 2002), barley genotypes with resistance to all evaluated races have also been identified. In greenhouse screening tests for barley stripe rust resistance using 25 isolates, accession PI 548740 ('Grannelose Zweizeilige' [GZ]) was one of eight lines resistant to all isolates (Chen et al. 1995). Other lines reported resistant to all of those isolates were 'Stauffers Obersulzer' (PI 467580), 'Abyssinian 14' (PI 151789), 'BBA 2890', 'Hor 1428' (PI 548708), 'Hor 2926' (PI 548734), 'Hor 3209' (PI

Corresponding author: K. Esvelt Klos; E-mail address: Kathy.klos@ars.usda.gov

http://dx.doi.org/10.1094/PHYTO-09-15-0225-R

This article is in the public domain and not copyrightable. It may be freely reprinted with customary crediting of the source. The American Phytopathological Society, 2016.
548747), and 'I 5' (PI 268187) (https://npgsweb.ars-grin.gov/gringlobal/ search.aspx). Segregation analyses suggested that resistance in these lines was mostly conditioned by recessive genes (Chen and Line 1999). Patterns of inheritance in progeny from the GZ $\times$ 'Steptoe' (Muir and Nilan 1973) cross suggested that resistance in GZ was due to a single recessive gene (Chen and Line 1999) but precise mapping and generation of reliable molecular markers was not possible in that study.

Some loci contributing to stripe rust resistance observed at the seedling stage have been mapped in barley. Quantitative trait loci (QTL) on chromosome $1 \mathrm{H}$ and $6 \mathrm{H}$ were mapped in the 'Shyri' $\times$ 'Galena' doubled-haploid population using 99 polymorphic markers (Castro et al. 2002; Toojinda et al. 2000). Seedling resistance in the $\mathrm{GZ} \times$ Steptoe population ( $r p s G Z$ ) was mapped to the long arm of chromosome $4 \mathrm{H}$ by comparing simple-sequence repeat banding patterns of recombinant inbred lines (RIL) with those of chromosome substitution lines (Yan and Chen 2006). Niks et al. (2015) conjectured that the major gene on chromosome $4 \mathrm{H}$ conferring resistance to $P$. striiformis f. sp. hordei (Rpsh) in the spring barley line 'L94' (Clho 11797) was the same as rpsGZ. A QTL on chromosome $4 \mathrm{H}$ contributing to field resistance, originally detected in the 'Calicuchima-Sib' × 'Bowman' (Franckowiak et al. 1985) mapping population ( $\log$ of the likelihood $[\mathrm{LOD}]=2.2$ ), also contributed to seedling resistance in a gene pyramiding population when present with the 1H QTL derived from Shyri (Castro et al. 2003b; Chen et al. 1994). Comparison of markers mapped in the Steptoe $\times$ GZ, L94 $\times$ 'Vada', and Calicuchima-Sib $\times$ Bowman populations against integrated maps in GrainGenes (http://wheat.pw.usda.gov) places these 4H QTL within an approximately 5-centimorgan (cM) region.

Marker-assisted selection (MAS) has been used to transfer stripe rust resistance QTL from unadapted source material into barley cultivars adapted for regional agronomic conditions (Toojinda et al. 1998). Markers have also been used to pyramid stripe rust resistance QTL derived from different sources into a single line (Castro et al. 2003a,b; Richardson et al. 2006). However, suitable single-nucleotide 
polymorphism (SNP) markers for the resistance QTL in GZ that can be used for high-throughput screening have not been developed. MAS would be particularly useful for transferring stripe rust resistance into elite germplasm from GZ, because GZ has very poor agronomic characteristics (P. Bregitzer, unpublished data), and multiple cycles of hybridization and selection will be necessary to develop cultivars with suitable performance.

The goal of the present study was to identify markers of the GZ stripe rust resistance for use in MAS. We refined the map locations and effect estimates of barley stripe rust resistance 4H QTL in GZ using a dense set of SNP; identified additional QTL on $4 \mathrm{H}, 6 \mathrm{H}$, and $7 \mathrm{H}$; and validated these markers for use in MAS by assessing their ability to predict disease resistance at the seedling stage in progeny from a different cross. The markers reported here may be used in future breeding efforts if polymorphic between parents, or may be used in conjunction with the publically available barley consensus maps to identify additional reliable markers for MAS.

\section{MATERIALS AND METHODS}

Plant materials. A population of 156 RIL was developed from a cross between GZ and 'Lenetah' barley by single-seed descent to the $\mathrm{F}_{5: 8}$ generation. GZ is a hull-less, awnless, two-rowed spring barley from Ethiopia. It is highly resistant to stripe rust but has exceptionally poor agronomic characteristics (P. Bregitzer, unpublished) (https://npgsweb.ars-grin.gov/gringlobal/search.aspx). Lenetah is a hulled, two-rowed spring feed barley with good agronomic characteristics developed by the United States Department of Agriculture-Agricultural Research Service (USDA-ARS) in Aberdeen, ID (Obert et al. 2008). A validation $\mathrm{F}_{5: 8}$ population of 165 RIL was developed using the same procedure from a cross of GZ with a two-rowed spring malting barley germplasm line '95SR316A' (Bregitzer et al. 2007).

Greenhouse and growth chamber screening. Lines from both mapping populations were assessed for seedling resistance to stripe rust in three trials as follows. Seed of five replicates of each parent, four replicates of $F_{1}$, and two replicates of each of the $F_{5: 8}$ RIL were sown in 2.5 -cm-diameter cone-tainers (Stuewe and Sons) filled with a vermiculite/peat moss/sand mixture in a 1:1:1 ratio by volume and Osmocote 15-9-12 slow release fertilizer (25 g/liter; Scotts). Plants were grown to the two-leaf stage in a greenhouse with a diurnal temperature setting of 12 to $25^{\circ} \mathrm{C}$ and a $14-\mathrm{h}$ photoperiod. Seedlings from all treatments were randomized with respect to their positions on the greenhouse bench and inoculated with freshly harvested urediniospores of North American race PSH54 [virulent on 'Topper', 'Abed Binder' [PI 662790], and 'Trumpf' [PI 548762]) and Bancroft (Wesenberg et al. 2001)] mixed with talc at a ratio of 1:100. Inoculated seedlings were placed in a dark dew chamber set to $12^{\circ} \mathrm{C}$ with $100 \%$ humidity for $24 \mathrm{~h}$. To check spore viability, a sample of the inoculum mixture was collected on water agar, incubated in the dew chamber alongside the plants, and rated for spore germination after $24 \mathrm{~h}$. After dew chamber treatment, the seedlings were transferred to growth chambers set to gradually ramp from $4^{\circ} \mathrm{C}$ during darkness to $20^{\circ} \mathrm{C}$ during light with a $16-\mathrm{h}$ photoperiod (Chen and Line 1992). After 18 to 22 days, infection type (IT) was rated on a 0 -to- 9 scale, in which $0=$ no visible signs or symptoms, $1=$ necrotic or chlorotic flecks with no sporulation, $2=$ necrotic or chlorotic blotches with no sporulation, $3=$ necrotic or chlorotic blotches with trace sporulation, $4=$ necrotic or chlorotic blotches with light sporulation, $5=$ necrotic or chlorotic blotches with intermediate sporulation, $6=$ necrotic or chlorotic blotches with moderate sporulation, $7=$ necrotic or chlorotic blotches with abundant sporulation, $8=$ chlorosis behind the sporulating area with abundant sporulation, and $9=$ no necrosis or chlorosis with abundant sporulation (Line and Qayoum 1992). For the purposes of posthoc analyses, RIL with mean IT ratings $<4$ were categorized as resistant (R), ratings $\geq 4$ to $<7$ as moderately resistant (MR), and ratings of $\geq 7$ as susceptible (S), concordant with McNeal et al.
(1971). To confirm P. striiformis f. sp. hordei race identity, the North American barley differentials (Chen et al. 1995) were grown, inoculated, and rated with the experimental materials in each of the three trials.

Field screening. Adult plant response to barley stripe rust, expressed as a percentage of leaf area infected (\%BSR), was assessed in the field in Corvallis, OR, and Mount Vernon, WA in 2013, 2014, and 2015; and in Davis, CA in the 2012-13 and 2014-15 field seasons. Data from field location/years was evaluated for quality prior to analysis and not used if stripe rust scores were influenced by nonuniform infection or high incidence of leaf rust. Based on these criteria, disease ratings used in analyses were from Corvallis in 2013 and 2015. In Corvallis 2013, single-row plots of RIL were planted with nine replicates of the parent varieties. In Corvallis 2015, single-row plots were planted in a randomized complete block design with two replications, including three replicates of the parent varieties within each replication. The 2015 data were analyzed as the mean response. Plots were bordered by spreader rows composed of susceptible 'Robust', 'Excel', 'Quest', 'Stander', and 'Rasmussen' barley.

Genotyping. DNA was extracted from leaf tissue of greenhousegrown plants using a cetyltrimethylammonium bromide (CTAB)based extraction buffer. In brief, seedling leaf tissue was freeze dried, ground, and incubated with $1 \mathrm{ml}$ of extraction buffer $(0.35 \mathrm{M}$ sorbitol, 0.3 M TrisHCl [pH 8.0], 5 mMEDTA [pH 8.0], $2 \mathrm{M} \mathrm{NaCl}, 2 \%$ CTAB, 5\% [wt/vol] N-lauroylsarcosine, $2 \%$ [wt/vol] polyvinylpyrrolidone [PVP40, K29-32], and 0.5\% [wt/vol] sodium metabisulfite) at $65^{\circ} \mathrm{C}$ for $1 \mathrm{~h}$. This aqueous mixture was combined with $24: 1$ chloroform/isoamyl alcohol at a 1:1 ratio, mixed, and centrifuged. The aqueous layer was removed and mixed with isopropanol at a 1:1 ratio. The precipitated DNA was pelleted by centrifugation, and the pellet was washed with $70 \% \mathrm{EtOH}$, dried, and resuspended in $10 \mathrm{mM}$ Tris buffer ( $\mathrm{pH}$ 8.5). Genotyping was conducted using the Infinium iSelect barley SNP array, as described by Muñoz-Amatriaín et al. (2014). SNP genotype calls were made with GenomeStudio (version 2011.11; Illumina). Monomorphic SNP and those with poor genotype calls due to weak signal or ambiguous clustering were eliminated. We used only the 1,882 polymorphic markers with map locations on the barley consensus map of Muñoz-Amatriaín et al. (2014).

For each statistically significant QTL, the 1-LOD confidence region around the peak LOD was identified. The expressed sequence tag sequence (available online at The Triticae Toolbox [T3]: https:// triticeaetoolbox.org/barley/) surrounding each SNP within the confidence region was examined. Five to six SNP per QTL were prioritized for assay design based on position with respect to the QTL (near the edges of the 1-LOD confidence interval and proximity to the peak LOD). Polymerase chain reaction (PCR) primers were designed that would amplify short sequences, including the SNP. The amplified sequences were subjected to real-time (RT)PCR-based high-resolution melt-curve assays designed to detect SNP within the amplicons. In this genotyping method, the average melting temperatures $\left(\mathrm{T}_{\mathrm{m}}\right)$ of PCR products are used to distinguish between amplicons that differ by a single nucleotide. Amplicons containing different SNP bases can vary by 0.4 to $0.6^{\circ} \mathrm{C}$. RT-PCR assays were conducted on a Bio-Rad CFX-96 using the following protocol: an initial denaturation of $98^{\circ} \mathrm{C}$ for $2 \mathrm{~min}$, followed by 35 cycles of denaturing at $98^{\circ} \mathrm{C}$ and annealing at $59.9^{\circ} \mathrm{C}$, followed by a melt-curve step ramping from $75^{\circ} \mathrm{C}$ to $90^{\circ} \mathrm{C}$ in $0.1^{\circ} \mathrm{C}$ increments for $5 \mathrm{~s}$ per increment. All reactions were completed in a final reaction volume of $20 \mu \mathrm{l}$ containing $10 \mu \mathrm{l}$ of SsoFast EvaGreen supermix (Bio-Rad), $600 \mathrm{nM}$ each primer, $6 \mu \mathrm{l}$ of sterile water purified by reverse osmosis, and $40 \mathrm{ng}$ of genomic DNA.

Statistical analysis. Greenhouse IT ratings of seedling resistance were evaluated using both the within-experiment scores and the mean score across experiments as the dependent variables. Field ratings were based on the \%BSR and evaluated within location/year. Composite interval mapping was performed using 
JMP Genomics v.7 (SAS Institute). Deviations from a normal distribution can increase the false-positive rate under this method. However, our intent was to seek validation of potential QTL in a second population. Therefore, we used 1,000 permutations to estimate an empirical LOD threshold necessary to achieve an $\alpha=$ 0.05 , and also chose to consider an LOD score $>2.0$ as suggestive (Morton 1998) and sufficient for advancement for analysis in the second population.

Other statistical analyses were performed in SAS Enterprise Guide 6.1 (SAS Institute) using the log-transformed IT scores, where appropriate. The number of lines in each category carrying GZ alleles at the QTL of interest was counted. Differences in mean $\%$ BSR field scores of lines that had been rated as R, MR, and S on the basis of seedling IT score were evaluated using a one-way analysis of variance. A Welch's test was used where variances were

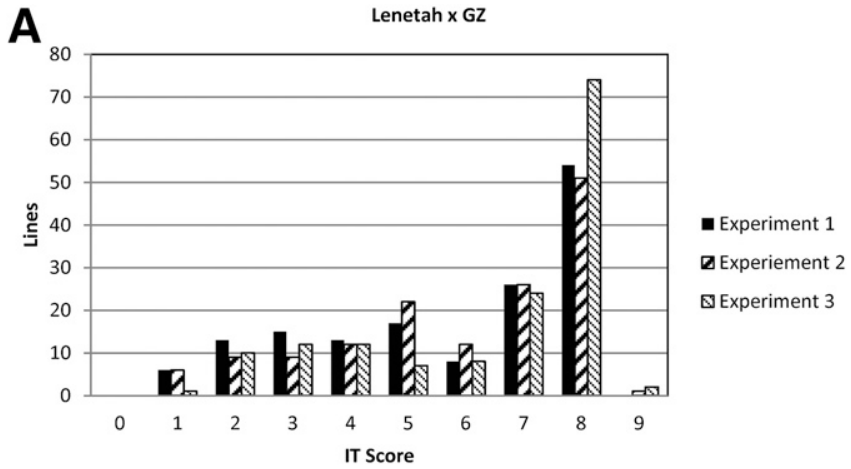

B

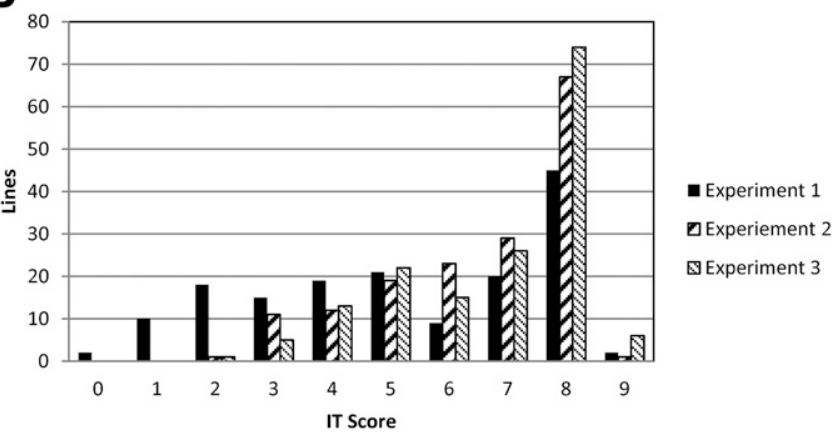

Fig. 1. Phenotypic distribution of infection type (IT) in recombinant inbred lines (RIL) of the A, Lenetah $\times$ Grannelose Zweizeilige (GZ) and B, 95SR316A $\times$ GZ RIL mapping populations when inoculated at the seedling stage with Puccinia striiformis $\mathrm{f}$. sp. hordei race $\mathrm{PSH}-54$. unequal based on the Levene's test. QTL effects on mean disease score at the seedling stage were evaluated using a linear regression approach similar to that of Castro et al. (2002), in which genotype data spanning a 1-LOD confidence interval around each QTL were used to infer parental haplotype for each RIL, and haplotype identity was used to test QTL effects in a factorial design. RIL with discernible crossover events in the QTL 1-LOD confidence intervals were set to missing in these analyses. RIL with undetectable double crossover events between marker and QTL remained in the analyses. Any such events would downwardly bias effect estimates. An $F$ statistic was used to evaluate the significance of effects by comparing nested models (a model that incorporates a given QTL effect compared with one that includes all effects in the first model except the QTL of interest).

\section{RESULTS}

The Lenetah $\times \mathbf{G Z}$ population. All four replicate $\mathrm{F}_{1}$ were $\mathrm{S}$, with a mean IT of 9 , indicating recessive inheritance of resistance from GZ. The IT score distributions of RIL from the seedling experiments were strongly skewed toward susceptible progeny, suggesting that more than one QTL was required for resistance (Fig. 1A). There was considerable plant-to-plant variability for IT score among GZ plants, with scores ranging from 0 to 5.5. However, the average IT score for GZ was 1.57, at the extreme lower end of the observed distribution. IT scores among Lenetah plants ranged from 7 to 9 , except for one plant in the second experiment scored as IT $=6$. The average IT score for Lenetah was 8.20. There was no evidence of transgressive segregation.

QTL mapping results were consistent among the three experiments; therefore, the mean IT ratings were used in subsequent analyses. Four QTL for seedling resistance were identified: two on chromosome $4 \mathrm{H}$ and one each on chromosomes $6 \mathrm{H}$ and $7 \mathrm{H}$ (Table 1 ; Fig. 2). The QTL with the highest LOD score (LOD = 15.94) was at $97.19 \mathrm{cM}$ on chromosome $4 \mathrm{H}$, designated $Q p s h 4 H b$. The empirical LOD threshold was determined to be LOD $\geq 2.5$.

To assess the degree of concordance between field and seedling resistance assays, the RIL were grouped based on resistance categories (R, MR, S) from the seedling experiments. Mean field response among groups was a significant source of variation $(P<$ 0.0001 ) (Table 2), indicating that both seedling and field resistance assays resulted in similar outcomes. In both years, lines considered $\mathrm{S}$ at the seedling stage had significantly higher \%BSR ratings as adult plants in the field than did lines rated as MR in seedling tests. Similarly, MR lines had higher \%BSR than R lines, although the contrast was statistically significant only in $2013(P<0.05)$. Consistent with these results, LOD scores from analyses of adult plant resistance were similar to those from analyses of seedling response

TABLE 1. Summary of quantitative trait loci (QTL) conferring resistance to Puccinia striiformis f. sp. hordei

\begin{tabular}{|c|c|c|c|c|c|c|c|}
\hline QTL & $\mathrm{Chr}^{\mathrm{a}}$ & $\mathrm{cM}^{\mathrm{b}}$ & 1-LOD interval ${ }^{c}$ & LOD & Additive effect ${ }^{\mathrm{d}}$ & $\operatorname{Var}(\%)^{\mathrm{e}}$ & Experiment $^{\mathrm{f}}$ \\
\hline Qpsh4Ha & $4 \mathrm{H}$ & 72.7 & $53.87-78.46$ & 5.39 & -1.44 & 15 & Seedling \\
\hline Qpsh4Hb & $4 \mathrm{H}$ & 97.19 & $94.84-102.18$ & 15.94 & -2.56 & 38 & Seedling \\
\hline Qpsh4Hb & $4 \mathrm{H}$ & 100.68 & $94.84-103.38$ & 8.72 & -19.85 & 23 & OR2013 \\
\hline Qpsh4Hb & $4 \mathrm{H}$ & 100.68 & $94.84-103.38$ & 4.43 & -18.13 & 12 & OR2015 \\
\hline Qpsh6H & $6 \mathrm{H}$ & 34.46 & $20.36-44.91$ & 4.24 & -1.44 & 12 & Seedling \\
\hline Qpsh6H & $6 \mathrm{H}$ & 27.19 & $20.36-42.43$ & 6.80 & -21.97 & 18 & OR2013 \\
\hline Qpsh6H & $6 \mathrm{H}$ & 27.19 & $20.36-42.43$ & 6.25 & -18.93 & 17 & OR2015 \\
\hline Qpsh7H & $7 \mathrm{H}$ & 81.07 & $76.06-82.23$ & 2.04 & -1.00 & 6 & Seedling \\
\hline Qpsh7H & $7 \mathrm{H}$ & 82.16 & $74.84-91.73$ & 3.62 & -22.73 & 10 & OR2013 \\
\hline Qpsh7H & $7 \mathrm{H}$ & 82.16 & $74.84-83.79$ & 6.45 & -14.36 & 18 & OR2015 \\
\hline
\end{tabular}

a Chromosome.

b Position of the peak marker in centimorgans (cM) on the consensus map of Muñoz-Amatriaín et al. (2014).

c Positions of markers flanking the peak with log of the likelihood (LOD) scores of (peak - 1).

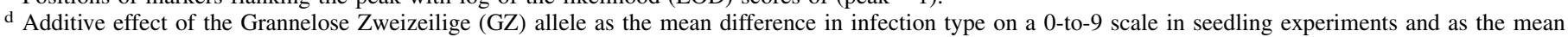
difference in percent severity in field experiments.

e Percentage of phenotypic variation (Var) accounted for by the QTL.

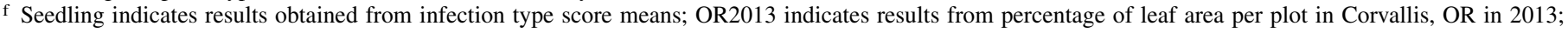
and OR2015 indicates results from percentage of leaf area per plot in Corvallis, OR in 2015. 
(Table 1; Fig. 2). The exception was $Q p s h 4 H a$, detected using seedling response under controlled conditions but not identified using adult plant response in either field experiment.

Markers within the 1-LOD confidence intervals around these QTL (Table 1) were used to classify RIL according to their probable allelic states. The nested linear regression model analyses supported the hypothesis of an additive genetic model, with models incorporating the effects of any given QTL region providing better predictive value than a reduced model without that region $(P<$ $0.01)$. IT mean values of Lenetah $\times$ GZ RIL that clearly possessed GZ alleles at these QTL (Fig. 3A) were generally in accord with \%BSR means (Fig. 3B). RIL with only GZ alleles at $6 \mathrm{H}$ had
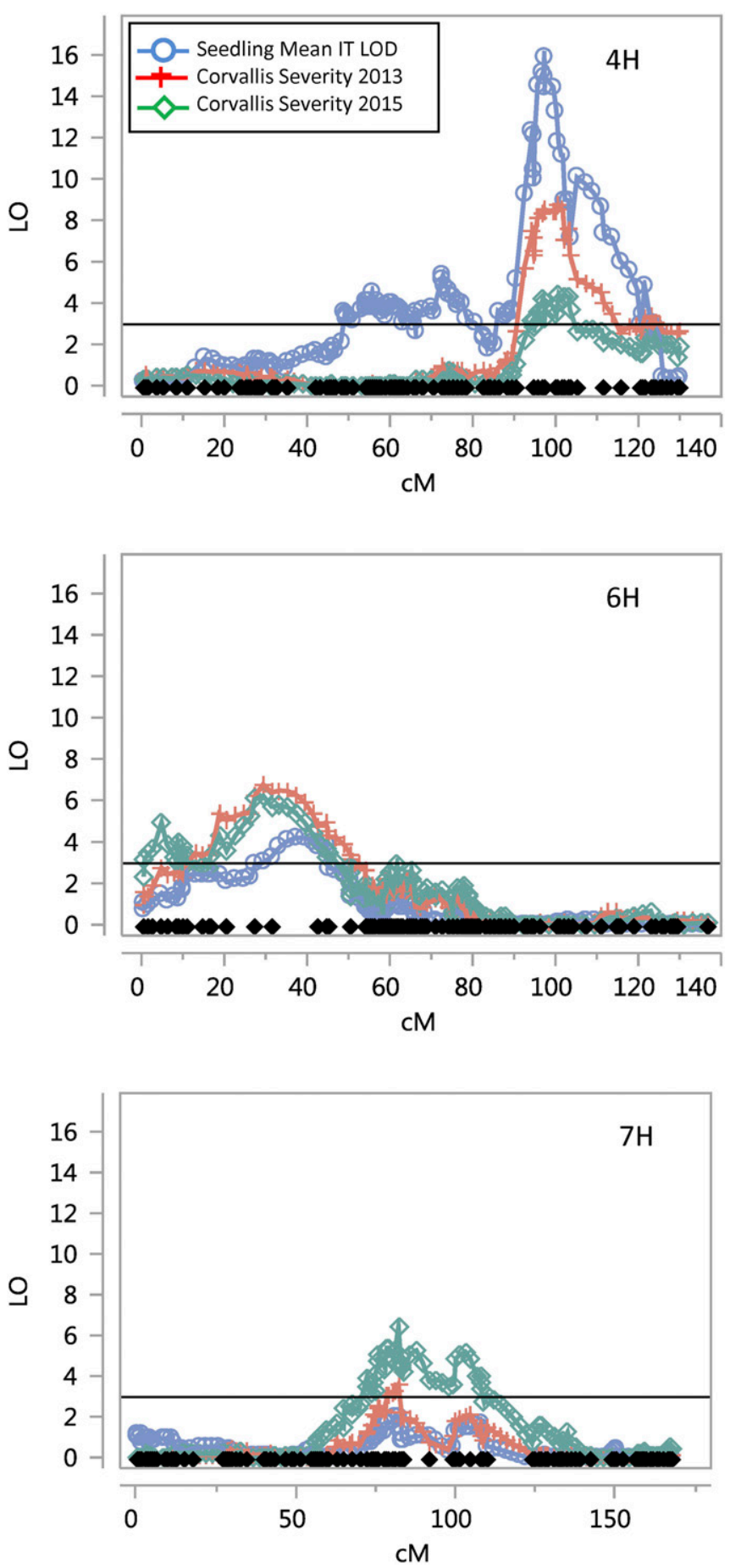

Fig. 2. Polymorphic marker map positions and log of the likelihood (LOD) scores from linkage analyses in the Lenetah $\times$ Grannelose Zweizeilige mapping population. The solid horizontal black line corresponds to LOD $=3.0$. unexpectedly low \%BSR, given that IT scores of that group did not differ from that of RIL carrying all Lenetah (S) alleles. In all, 11 of 12 resistant RIL had the GZ (R) alleles at $4 \mathrm{Hb}, 6 \mathrm{H}$, and at least one other QTL (Table 3), and overall RIL with that pattern of inheritance had lower mean IT scores than did other RIL $(P<0.01)$. In addition, lines in those genotype categories had mean BSR $<11 \%$ in both years (Fig. 3B).

SNP markers within the 1-LOD confidence interval were selected as candidates for use in MAS. Genotype calls from the highresolution melt curve and Illumina platform for the Lenetah $\times \mathrm{GZ}$ RIL were $100 \%$ consistent for markers in the $4 \mathrm{Ha}, 6 \mathrm{H}$, and $7 \mathrm{H}$ QTL regions. The SCRI_RS_188827,SCRI_RS_10818, and SCRI_RS_ 157611 markers in the $4 \mathrm{Hb}$ region exhibited $7.1,0.6$, and $1.3 \%$ inconsistency, respectively, between melt-curve and Illumina genotype calls, based on all RIL. Using the melt-curve assay genotypes instead of the full set of Illumina genotypes to assign QTL allelic states resulted in reclassification of some affected lines as having crossover events rather than one or the other parental allele.

The 95SR316A $\times$ GZ population. Seedling IT scores for this population were used to validate the results obtained from analyses of the Lenetah $\times \mathrm{GZ}$ population. The distribution of seedling resistance IT scores among progeny of $95 \mathrm{SR} 316 \mathrm{~A} \times \mathrm{GZ}$ was similar to that of Lenetah $\times$ GZ (Fig. 1B). The four F1 plants tested had IT scores of $9(\mathrm{~S})$, as expected for recessive inheritance. The mean IT scores for GZ and 93SR316A were 3.4 and 7.3, respectively.

RIL of the 95 SR $316 \mathrm{~A} \times \mathrm{GZ}$ population were genotyped via highresolution melt-curve assay only for the $12 \mathrm{SNP}$ corresponding to the $4 \mathrm{Ha}, 4 \mathrm{Hb}, 6 \mathrm{H}$, and 7H QTL (Table 4). The nested linear regression model analyses of IT score in the $95 \mathrm{SR} 316 \mathrm{~A} \times \mathrm{GZ}$ population also supported the hypothesis of an additive genetic model, with models incorporating the effects of $4 \mathrm{Ha}, 4 \mathrm{Hb}$, and $6 \mathrm{H} \mathrm{QTL}$ regions providing greater predictive value than a reduced model without that region $(P<0.01)$. The nested model analyses did not support an independent effect for the 7H QTL $(P>0.01)$ in this population. Mean IT scores of RIL with the $4 \mathrm{Hb}$ QTL in combination with at least one other QTL were lower than those of other RIL $(P<0.01$; Fig. 4). As with the Lenetah $\times$ GZ populations, resistant 95SR316A $\times$ GZ RIL all possessed the $4 \mathrm{Hb}$ QTL but not all lines possessing the GZ allele at $4 \mathrm{Hb}$ were resistant (Table 3 ).

\section{DISCUSSION}

Composite interval mapping in the Lenetah $\times$ GZ RIL identified QTL on chromosome $4 \mathrm{H}$ and $6 \mathrm{H}$ influencing seedling reaction to infection with race $\mathrm{PSH}-54$, and suggested the presence of an additional QTL on chromosome 7H. The 4H QTL ( Qpsh4Hb in this study) previously identified by Yan and Chen (2006) was confirmed. The finding that GZ possesses multiple QTL conferring resistance to $P$. striiformis $\mathrm{f}$. sp. hordei is a departure from previous studies. The original survey of 31 barley genotypes by Chen et al.

TABLE 2. Field responses of Lenetah $\times$ Grannelose Zweizeilige (GZ) recombinant inbred lines to barley stripe rust infection in Corvallis, OR in 2013 and $2015^{\mathrm{a}}$

\begin{tabular}{|c|c|c|c|c|c|c|c|}
\hline \multirow[b]{2}{*}{ Resistance } & \multirow[b]{2}{*}{$N$} & \multicolumn{3}{|c|}{ \%BSR 2013} & \multicolumn{3}{|c|}{ \%BSR 2015} \\
\hline & & Mean & $\mathrm{SD}$ & Range & Mean & $\mathrm{SD}$ & Range \\
\hline $\mathrm{R}$ & 26 & 7.9 & 9.6 & $0-30$ & 9.9 & 13.6 & $0-45$ \\
\hline MR & 53 & 19.7 & 12.5 & $0-55$ & 18.7 & 18.4 & $0-65$ \\
\hline $\mathrm{S}$ & 77 & 36.9 & 21.4 & $5-80$ & 39.4 & 25.9 & $0-90$ \\
\hline All & 156 & 28.0 & 22.8 & $0-80$ & 27.6 & 26.4 & $0-90$ \\
\hline
\end{tabular}

a Data are presented as the mean percentage of affected leaf area (BSR\%) and broken down by seedling response groups. $\mathrm{SD}=$ standard deviation. Mean difference among resistance categories is statistically significant $(P<$ $0.0001)$.

${ }^{\mathrm{b}}$ Resistance was categorized based on seedling infection type score as $\mathrm{R}$ $($ resistant $) \leq 4.0, \mathrm{MR}($ moderate reaction $)=4.0$ to 6.9 , and $\mathrm{S}$ (susceptible $) \geq 7.0$. 
(1995) found that GZ was among those resistant to all 25 P. striiformis f. sp. hordei isolates evaluated. Segregation analyses of $F_{2}$ seedlings and $F_{3}$ families from a cross between Steptoe and GZ were used to hypothesize the presence of a major recessive gene conferring resistance to $P$. striiformis f. sp. hordei in GZ (Chen and Line 1999). This gene was subsequently mapped to chromosome $4 \mathrm{H}$ using RIL derived from the Steptoe $\times$ GZ cross screened with races PSH-14 and PSH-54 (Yan and Chen 2006).

The hypothesis of a single recessive gene conferring resistance in GZ was made on the basis of goodness-of-fit to a 1:3 R/S ratio in the $\mathrm{F}_{2}$ and a 1:2:1 R/segregating/S ratio in $\mathrm{F}_{3}$ families (Chen and Line 1999). However, the methods used by Yan and Chen (2006) for follow-up mapping in the Steptoe $\times$ GZ population would not have been capable of identifying the $4 \mathrm{Ha}, 6 \mathrm{H}$, and $7 \mathrm{H}$ QTL. Because of limited marker availability at that time, a bulked segregant approach was used to identify markers for mapping. First, markers were selected based on their ability to differentiate a bulk of resistant RIL from a bulk of susceptible RIL, with reference to the parent genotypes. At this stage, markers linked to all four QTL may have been selected. At the second stage, the selected markers were screened for consistency across resistant and susceptible RIL. In the current mapping population, we found only the $4 \mathrm{Hb}$ QTL consistently present in resistant RIL. Thus, assuming the same inheritance in Steptoe $\times$ GZ, the markers retained by Yan and Chen (2006) following their second screening step would have been those linked to the $4 \mathrm{Hb}$ QTL but (perhaps coincidentally) not present in

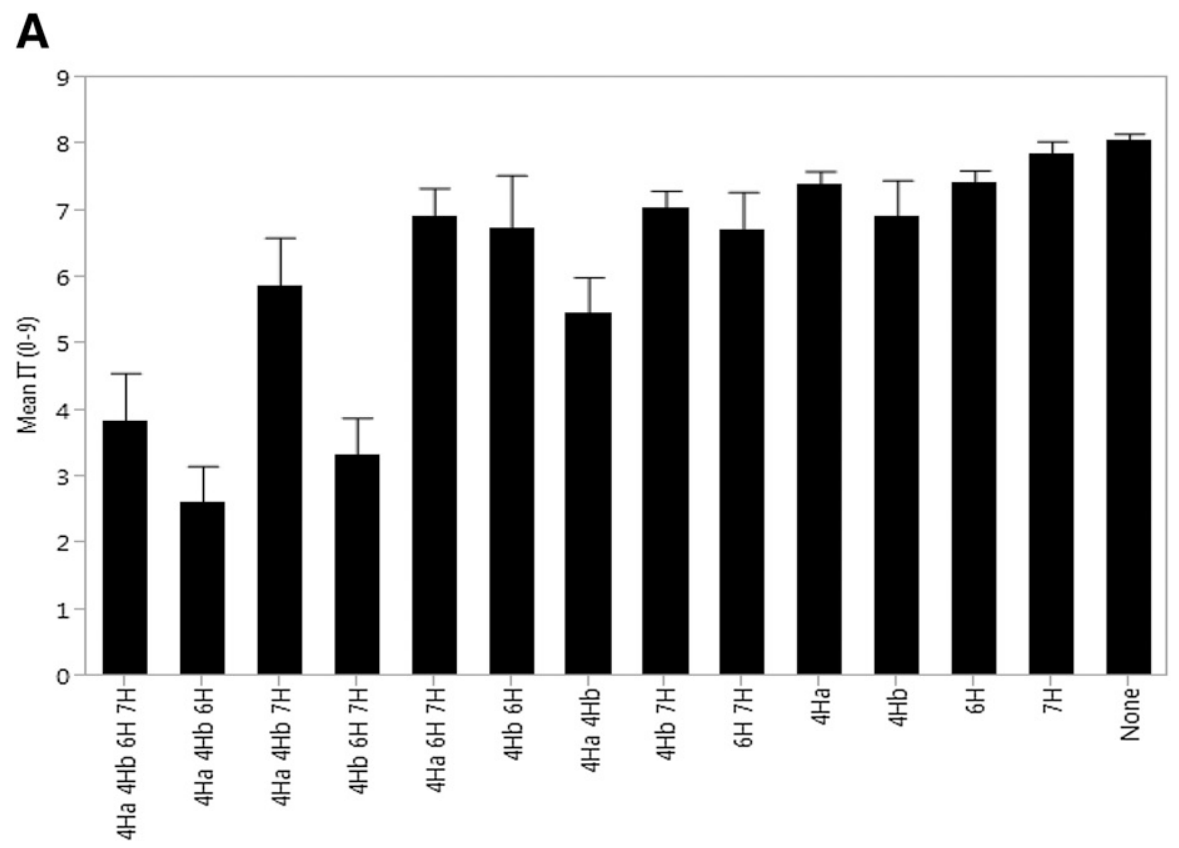

B

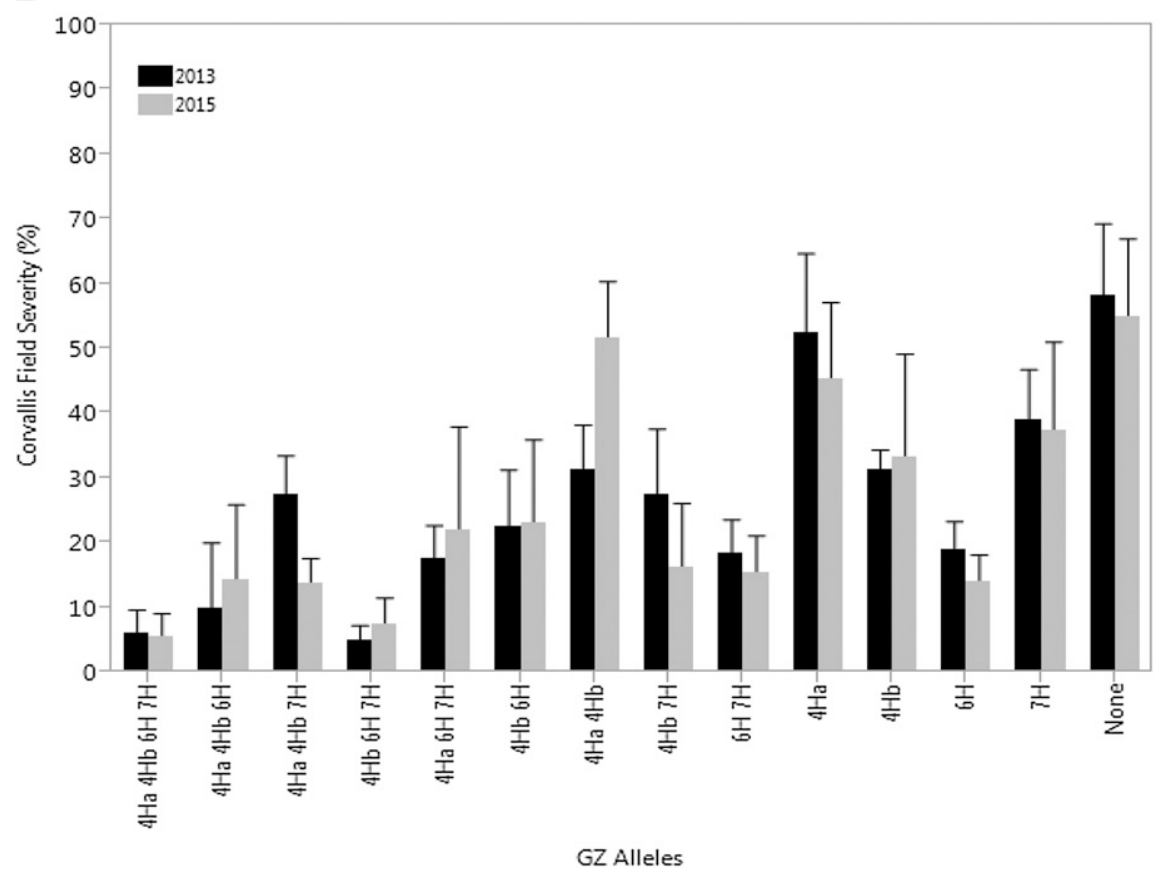

Fig. 3. Mean infection type scores from $\mathbf{A}$, seedling experiments and $\mathbf{B}$, adult plant reactions from field trials for recombinant inbred lines of the Lenetah $\times$ Grannelose Zweizeilige (GZ) population that have GZ-derived alleles at quantitative trait loci on $4 \mathrm{H}$ at $55.59 \mathrm{cM}(4 \mathrm{Ha}), 4 \mathrm{H} \mathrm{at} 97.19 \mathrm{cM}(4 \mathrm{Hb}), 6 \mathrm{H}$ at $31.19 \mathrm{cM}$ $(6 \mathrm{H})$, and $7 \mathrm{H}$ at $81.07 \mathrm{cM}(7 \mathrm{H})$. Genotype classes represented by fewer than three lines are not shown. Bars indicate the standard error. 
any susceptible RIL. Substantial advances in genotyping technology and linkage map density have made the current mapping methods possible, in which no assumptions were made concerning mode of inheritance or number of contributing loci.

Alternately, the Steptoe $\times$ GZ population may have been segregating at only the single QTL (designated rpsGZ), with one or more minor genes fixed in that population. Support for this hypothesis comes from a recently published QTL map using RIL from a cross between the Ethiopian line L94 and Vada, developed in the Netherlands (Niks et al. 2015). In that cross, major gene Rpsh was mapped to 96.17 to $98.33 \mathrm{cM}$ on the Barley, Consensus 2006, Marcel map on the GrainGenes website (http://wheat.pw. usda.gov). Given the large effect size and the chromosomal position, the authors concluded that this gene was most likely rpsGZ.

An argument in favor of the multigenic hypothesis for GZ resistance is the consistency of mapping results between controlledenvironment and field studies. We assessed IT at the seedling stage under controlled conditions, and disease severity based on the 0 to $100 \%$ scale in the field. IT summarized plant reactions to the pathogen through a single infection cycle, while severity summarized resistance throughout the rust season over multiple infection cycles. LOD peaks from analyses of field response to $P$. striiformis f. sp. horde $i$ were consistent with the location of those for seedling IT score. Lower disease ratings, both seedling IT and field \%BSR, were

TABLE 3. Number of lines of the Lenetah $\times$ Grannelose Zweizeilige (GZ) and 95SR316A $\times$ GZ mapping populations classified for barley stripe rust resistance based on seedling tests and deemed to possess GZ-derived alleles at regions defined by markers flanking four quantitative trait loci (QTL) ${ }^{\mathrm{a}}$

\begin{tabular}{|c|c|c|c|c|c|c|c|c|c|}
\hline \multicolumn{4}{|c|}{ QTL locations } & \multicolumn{3}{|c|}{ Lenetah $\times \mathrm{GZ}$} & \multicolumn{3}{|c|}{$95 \mathrm{SR} 316 \mathrm{~A} \times \mathrm{GZ}$} \\
\hline $4 \mathrm{Ha}$ & $4 \mathrm{Hb}$ & $6 \mathrm{H}$ & $7 \mathrm{H}$ & $\mathrm{R}$ & MR & $\bar{S}$ & $\mathrm{R}$ & MR & $S$ \\
\hline $\mathrm{GZ}$ & GZ & GZ & $\mathrm{GZ}$ & 3 & 2 & 0 & 0 & 4 & 1 \\
\hline$\ldots$ & GZ & $\mathrm{GZ}$ & $\mathrm{GZ}$ & 4 & 1 & 0 & 2 & 5 & 1 \\
\hline $\mathrm{GZ}$ & $\ldots$ & $\mathrm{GZ}$ & $\mathrm{GZ}$ & 0 & 3 & 1 & 0 & 1 & 0 \\
\hline $\mathrm{GZ}$ & GZ & $\ldots$ & $\mathrm{GZ}$ & 0 & 6 & 2 & 0 & 5 & 0 \\
\hline $\mathrm{GZ}$ & GZ & $\mathrm{GZ}$ & $\ldots$ & 4 & 0 & 0 & 2 & 1 & 0 \\
\hline$\ldots$ & $\ldots$ & $\mathrm{GZ}$ & $\mathrm{GZ}$ & 0 & 1 & 3 & 0 & 2 & 3 \\
\hline GZ & $\ldots$ & $\ldots$ & $\mathrm{GZ}$ & 0 & 0 & 2 & 0 & 2 & 2 \\
\hline $\mathrm{GZ}$ & $\ldots$ & $\mathrm{GZ}$ & $\ldots$ & 0 & 0 & 1 & 0 & 0 & 1 \\
\hline$\ldots$ & GZ & $\ldots$ & $\mathrm{GZ}$ & 0 & 1 & 3 & 1 & 4 & 1 \\
\hline$\ldots$ & GZ & GZ & $\ldots$ & 0 & 2 & 2 & 1 & 2 & 0 \\
\hline GZ & GZ & $\ldots$ & $\ldots$ & 1 & 4 & 1 & 2 & 5 & 1 \\
\hline$\ldots$ & $\ldots$ & $\ldots$ & GZ & 0 & 0 & 5 & 0 & 0 & 7 \\
\hline$\ldots$ & $\ldots$ & $\mathrm{GZ}$ & $\ldots$ & 0 & 0 & 5 & 0 & 1 & 5 \\
\hline$\ldots$ & $\mathrm{GZ}$ & $\ldots$ & $\ldots$ & 0 & 2 & 2 & 0 & 4 & 1 \\
\hline GZ & $\ldots$ & $\ldots$ & $\ldots$ & 0 & 1 & 5 & 0 & 2 & 5 \\
\hline$\ldots$ & $\ldots$ & $\ldots$ & $\ldots$ & 0 & 1 & 6 & 0 & 1 & 4 \\
\hline
\end{tabular}

${ }^{a} \mathrm{R}, \mathrm{MR}$, and $\mathrm{S}$ indicate mean infection type scores from seedling resistance tests of $<4, \geq 4$ to $<7$, and $\geq 7$, respectively. observed for lines carrying resistance alleles at $Q p s h 4 H b, Q p s h 6 H$, and one other QTL (Qpsh4Ha or $Q p s h 7 H)$ (Fig. 3). This remarkable consistency suggests that the high level of resistance in GZ is largely all-stage resistance, detected at the seedling stage as IT and in adult plants as both IT and severity. There were differences, however, in the relative effects of QTL in seedling versus field tests. Nonsignificant $(\mathrm{LOD}<2.0)$ peaks in the $4 \mathrm{Ha}$ region were detected in both 2013 and 2015, suggesting that this QTL is much less effective in the field against $P$. striiformis f. sp. hordei infection. Conversely, $Q p s h 7 H$ was statistically significant in analyses of field data in both years but not significant in analyses of seedling IT scores. The QTL detected here may have different mechanisms underlying their contributions to resistance. Further work is needed to dissect the mechanisms of action underlying specific QTL, perhaps using nearisogenic lines.

The 7H QTL (at 81.07 to $82.06 \mathrm{cM}$ ) was mapped to an overlapping location with the nud gene conditioning hull-lessness that was also contributed by GZ (LOD = 91.99 at $83.79 \mathrm{cM}$; not shown). Thus, the 7H QTL may be more difficult to transfer to elite barley germplasm without also transferring the hull-less trait.

It is interesting to compare the resistance of GZ with that from other germplasm sources. QTL on $5 \mathrm{H}$ and $6 \mathrm{H}$ donated by the tworow feed barley Shyri were identified in field studies of adult plant response and also in controlled-environment studies of seedling resistance (Castro et al. 2002; Toojinda et al. 2000). In those studies, lines with Shyri alleles at both QTL were more likely to be resistant in seedling tests and had lower average disease severity in the field. This result is similar to the performance of the GZ $6 \mathrm{H}$ and $4 \mathrm{Hb}$ QTL in the current study. Indeed, consensus maps available in GrainGenes (http://wheat.pw.usda.gov) place the GZ QTL on chromosome $6 \mathrm{H}$ in the same region as the Shyri QTL. Similarly, other mapping studies have identified $P$. striiformis $\mathrm{f}$. sp. hordei resistance on chromosome 4H. Extensive characterization was performed on the 4H QTL derived from Calicuchima-sib in combination with QTL on $1 \mathrm{H}, 5 \mathrm{H}$, and $6 \mathrm{H}$ derived from Calicuchima-sib and Shyri (Castro et al. 2003a,b; Chen et al. 1994; Toojinda et al. 1998). The effect of the 4H QTL was observed only in combination with the resistant allele at another QTL. A QTL on $4 \mathrm{H}$ influencing adult plant resistance at the same location was also mapped in progeny of the BCD47 (PI 659444) $\times$ Baronesse (PI 568246), which were segregating for QTL in six genomic regions, including on $6 \mathrm{H}$ and $7 \mathrm{H}$; however, there was no indication of epistatic interaction among QTL in that cross (Richardson et al. 2006; Vales et al. 2005).

SNP within the genomic region underlying $Q p s h 4 H b$ correspond to 97.31 to $99.22 \mathrm{cM}$ in the BARLEYMAP pipeline (Cantalapiedra et al. 2015) and 518.79 to $522.85 \mathrm{Mb}$ in the EnsemblPlants viewer (Kersey et al. 2014). This genomic region is annotated with over 100 genes, many of which are uncharacterized but several of which have potential as candidates for barley stripe rust resistance. The barley mlo gene maps to this region (Kersey et al. 2014) and is a strong

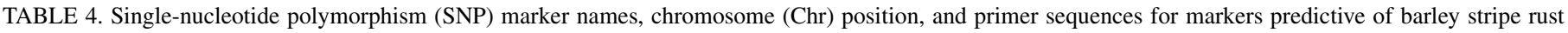
resistance derived from Grannelose Zweizeilige ${ }^{\mathrm{a}}$

\begin{tabular}{|c|c|c|c|c|}
\hline SNP & Chr & Location $(\mathrm{cM})^{\mathrm{b}}$ & Forward primer & Reverse primer \\
\hline SCRI_RS_190401 & $4 \mathrm{H}$ & 53.87 & GGTGGAATTGCAGACAGACC & GCCCTGGCAAGAAATAACAG \\
\hline BOPA_12_30839 & $4 \mathrm{H}$ & 54.95 & ССTGCAATCTTCTAAACTAGTCAGTG & AAGCATGCCATAGACAAATGAA \\
\hline SCRI_RS_-168603 & $4 \mathrm{H}$ & 55.59 & ACACCACACAGCGCACAGA & GTCGGCTTGGTTCGATTC \\
\hline SCRI_RS_188827 & $4 \mathrm{H}$ & 97.29 & GAAGTGCACGGTATGTTCTTGG & TCTCGCTTACAAGCATCTCTGC \\
\hline SCRI_RS_10﹎10 & $4 \mathrm{H}$ & 101.68 & CCGAAGCTAAATACGTCGCTCT & TAGGCAGCTTTGAAGTGAGCAG \\
\hline SCRI_RS_157611 & $4 \mathrm{H}$ & 103.38 & TGCATCTTCTCCGTCAGGTA & GCCTGCACCTTCATCGTAGT \\
\hline BOPĀ1_1240-844 & $6 \mathrm{H}$ & 20.36 & GGCCAAACTACCTGATGCTC & CGGGTAGTAGTCCACCTCCT \\
\hline SCRI_RS_189878 & $6 \mathrm{H}$ & 31.19 & GCTATGCCTACCCCCAACTT & GGCTGCTGCCTATGAATGAG \\
\hline BOPA2_12_30358 & $6 \mathrm{H}$ & 42.43 & CCGATTCTCGGTGAAGAAAA & CTTCTCGTGGAAGTAGGCTAGG \\
\hline BOPA1_478-1291 & $7 \mathrm{H}$ & 78.07 & ACATGGCCAAGACCCAGTAG & TAGCGACACCATCCACATTT \\
\hline SCRI_RS_235584 & $7 \mathrm{H}$ & 79.37 & CGATCCATCTCTTCCTCCTG & GTACACTGCCCCCACCTTG \\
\hline BOPĀ1_1272-459 & $7 \mathrm{H}$ & 81.07 & CAGGCTGCTCAAGGCCTAC & ATGTCCTCGTCCTCGAATTG \\
\hline
\end{tabular}

a SNP marker expressed sequence tag sequences are available online at The Triticae Toolbox (T3) (https://triticeaetoolbox.org/barley/).

b Centimorgan (cM) position on the consensus map of Muñoz-Amatriaín et al. (2014). 
candidate. MLO controls defense response to powdery mildew caused by Blumeria graminis f. sp. hordei, with recessive mutations conferring nonrace-specific resistance (Büschges et al. 1997; Peterhänsel and Lahaye 2005). For mlo to confer powdery mildew resistance, the action of cofactors is required (Peterhänsel and Lahaye 2005). This cofactor requirement is reminiscent of the GZ allele at the $4 \mathrm{Hb} \mathrm{QTL}$ observed in this study conferring resistance to $P$. striiformis f. sp. hordei. Recently, allelic variation at $m l o$ has been implicated in the relative susceptibility of barley to Ramularia leaf spot caused by the fungus Ramularia collo-cygni (McGrann et al. 2014), suggesting a role for this gene outside of powdery mildew resistance. Two MLO region markers were genotyped on the Lenetah $\times$ GZ population but neither was polymorphic between parents and, thus, did not segregate.

In addition to mlo, several other genes in the $Q p s h 4 H b$ region have been implicated in processes related to managing cellular reactive oxygen species, an important part of the plant defense response (Hammond-Kosack and Jones 1996; Low and Merida 1996). Homologs of catalase (MLOC_76546), respiratory burst oxidase protein (MLOC_55429), and peptide-methionine (R)-S-oxide reductase (MLOC_56572) are present in this region. In Arabidopsis, catalase mutants exhibit altered gene expression profiles following fungal infection (Mhamdi et al. 2010). Respiratory burst oxidase homologs play diverse roles in regulating plant response to the environment, including signaling defense responses to fungal pathogens (Suzuki et al. 2011). Study of peptide-methionine (R)$\mathrm{S}$-oxide reductase enzyme levels in poplar leaves revealed differential levels after compatible or incompatible reactions to Melampsora larici-populina (Dos Santos et al. 2005). The gene MLOC_8329, which also maps to this region, is likely a defensin homolog. Defensins are diverse proteins, expressed in all tissues but most abundantly in seed, which are involved in defense against a broad range of fungi (Stotz et al. 2009).
Critically, the current work indicates that markers for at least two QTL may be required for successful MAS of resistance derived from GZ. Although some elite barley lines may carry genes with the capacity to act as modifiers to $\operatorname{rps} G Z$, that was not the case in either cross used in this study. Markers of practical utility for breeders were identified, and the high-resolution melt-curve assays for SNP flanking and within each GZ-derived QTL are based on commonly available equipment and reagents. The reliability of detection of GZ alleles via high-resolution melt-curve assay was validated based on comparison with genotyping using the barley 9K Illumina SNP genotyping platform. The high-resolution melt-curve assays also enabled the detection of lines with crossover events within markerdefined regions.

In some circumstances, limited resources might make the use of a single marker per QTL preferable. The use of single SNP markers located within these QTL regions would have misclassified 0 to $17 \%$ of lines, depending on the marker, with a crossover event as carriers of a single parental allele across the relevant QTL regions. The proportion of lines erroneously retained for advancement in a breeding program during MAS would then depend on how close the selected marker was to the resistance genes underlying the QTL, a factor not deducible from the present data.

Had we used the markers presented here for selection of progeny based on carrier status at the $4 \mathrm{Hb}$ and $6 \mathrm{H}$ QTL, we would have retained 11 of $26 \mathrm{R}$ lines of the Lenetah $\times \mathrm{GZ}$ population and 6 of $18 \mathrm{R}$ lines of the $95 \mathrm{SR} 316 \mathrm{~A} \times \mathrm{GZ}$ population for advancement in the breeding program. We would also have retained 2 of $76 \mathrm{~S}$ and 14 of $55 \mathrm{MR}$ Lenetah $\times \mathrm{GZ}$ lines, and 3 of $79 \mathrm{~S}$ and 13 of 61 MR 95SR316A $\times$ GZ lines. Overall, resources in advanced trials would have been concentrated on $\mathrm{R}$ to MR lines, thus increasing the likelihood that lines selected for other agronomic or quality characteristics would also possess resistance to barley stripe rust.

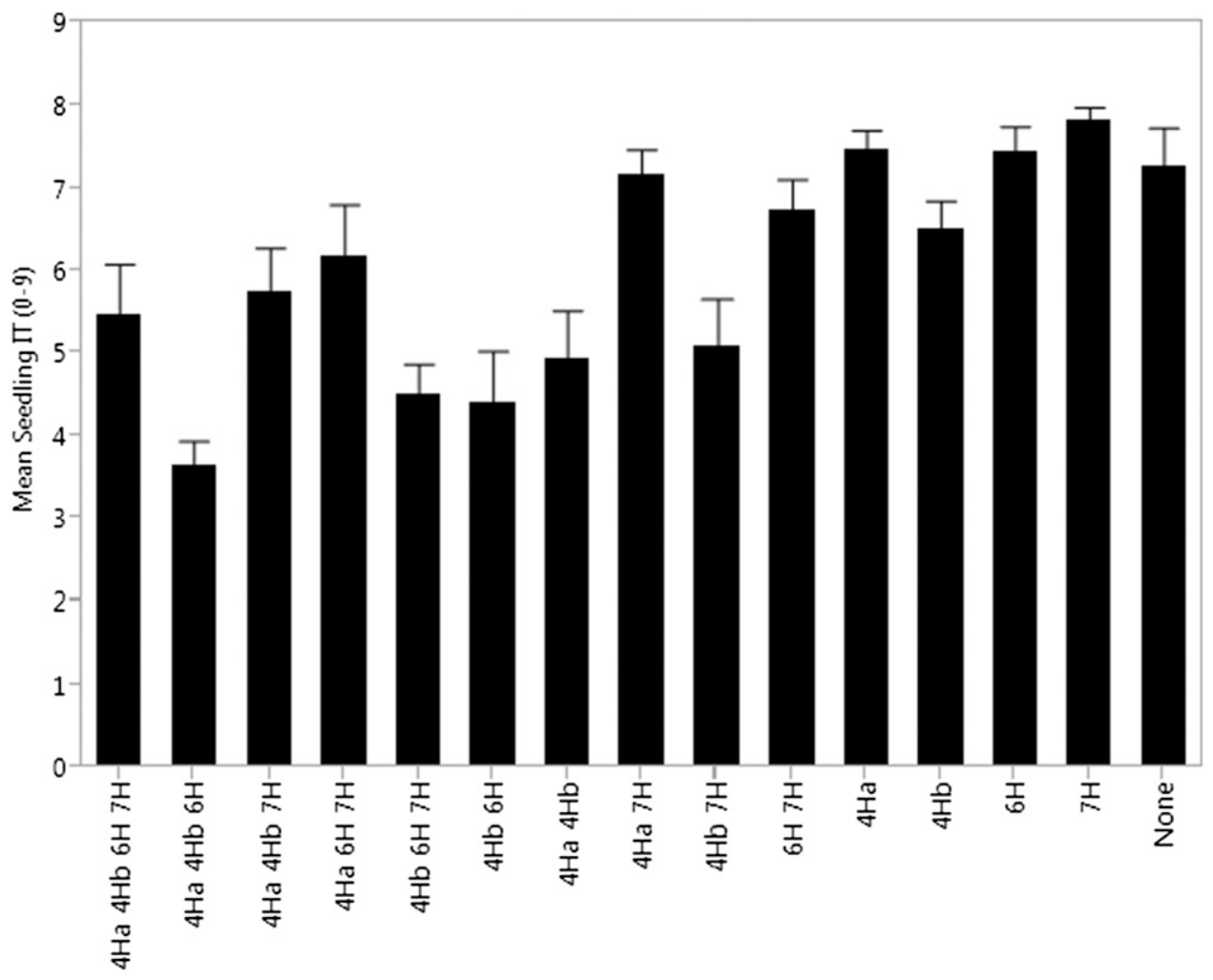

GZ Alleles

Fig. 4. Mean infection type scores from seedling experiments for recombinant inbred lines of the $95 \mathrm{SR} 316 \mathrm{~A} \times$ Grannelose Zweizeilige (GZ) population that have GZ-derived alleles at quantitative trait loci (QTL) on chromosomes $(\mathrm{Chr}) 4 \mathrm{H}$ at $55.59 \mathrm{cM}(4 \mathrm{Ha}), 4 \mathrm{H}$ at $97.19 \mathrm{cM}(4 \mathrm{Hb}), 6 \mathrm{H} \mathrm{at} 31.19 \mathrm{cM}(6 \mathrm{H})$, and $7 \mathrm{H}$ at $81.07 \mathrm{cM}$ $(7 \mathrm{H})$. Genotype classes represented by fewer than three lines are not shown. Bars indicate the standard error. 


\section{ACKNOWLEDGMENTS}

This work was supported by the United States Department of AgricultureAgricultural Research Service, Project Number 2050-21000-031-00. We thank R. Campbell and I. Shakelford for their technical assistance and J. Dubcovsky for his contribution to planning for field evaluations.

\section{LITERATURE CITED}

Bregitzer, P., Obert, D. E., and Wesenberg, D. M. 2007. Registration of 95SR316A stripe rust-resistant two-rowed malting barley germplasm. J. Plant Reg. 1:141-142.

Brown, W. M., Jr., Hill, J. P., and Velasco, V. R. 2001. Barley yellow rust in North America. Annu. Rev. Phytopathol. 39:367-384.

Büschges, R., Hollricher, K., Panstruga, R., Simons, G., Wolter, M., Frijters, A., van Daelen, R., van der Lee, T., Diergaarde, P., Groenendijk, J., Töpsch, S., Vos, P., Salamini, F., and Schulze-Lefert, P. 1997. The barley Mlo gene: A novel control element of plant pathogen resistance. Cell 88:695-705.

Cantalapiedra, C. P., Boudiar, R., Casas, A. M., Igartua, E., and ContrerasMoreira, B. 2015. BARLEYMAP: Physical and genetic mapping of nucleotide sequences and annotation of surrounding loci in barley. Mol. Breed. 35:13.

Castro, A. J., Chen, X. M., Corey, A., Filichkina, T., Hayes, P. M., Mundt, C., Richardson, K., Sandoval-Islas, S., and Vivar, H. 2003a. Pyramiding and validation of quantitative trait locus (QTL) alleles determining resistance to barley stripe rust: Effects on adult plant resistance. Crop Sci. 43:2232-2239.

Castro, A. J., Chen, X. M., Hayes, P. M., and Johnston, M. 2003b. Pyramiding quantitative trait locus (QTL) alleles determining resistance to barley stripe rust: Effects on resistance at the seedling stage. Crop Sci. 43:651-659.

Castro, A. J., Chen, X. M., Hayes, P. M., Knapp, S. J., Line, R. F., Toojinda, T., and Vivar, H. 2002. Coincident QTL which determine seedling and adult plant resistance to stripe rust in barley. Crop Sci. 42:1701-1708.

Chen, F. Q., Prehn, D., Hayes, P., Mulrooney, D., Corey, A., and Vivar, H. 1994. Mapping genes for resistance to barley stripe rust (Puccinia striiformis f. sp. hordei). Theor. Appl. Genet. 88:215-219.

Chen, X. M., and Line, R. F. 1992. Inheritance of stripe rust resistance in wheat cultivars used to differentiate races of Puccinia striiformis in North America. Phytopathology 82:633-637.

Chen, X. M., and Line, R. F. 1999. Recessive genes for resistance to Puccinia striiformis f. sp. hordei in barley. Phytopathology 89:226-232.

Chen, X. M., Line, R. F., and Leung, H. 1995. Virulence and polymorphic DNA relationships of Puccinia striiformis f. sp. hordei to other rusts. Phytopathology 85:1335-1342.

Dos Santos, C. V., Cuiné, S., Rouhier, N., and Rey, P. 2005. The Arabidopsis plastidic methionine sulfoxide reductase B proteins. Sequence and activity characteristics, comparison of the expression with plastidic methionine sulfoxide reductase A, and induction by photooxidative stress. Plant Physiol. 138:909-922.

Dubin, H. J., and Stubbs, R. W. 1986. Epidemic spread of barley stripe rust in South America. Plant Dis. 70:141-144.

Franckowiak, J. D., Foster, A. E., Pederson, V. D., and Pyler, R. E. 1985. Registration of Bowman barley. Crop Sci. 25:883.

Hammond-Kosack, K. E., and Jones, D. G. 1996. Resistance gene-dependent plant defense responses. Plant Cell 8:1773-1791.

Kersey, P. J., Allen, J. E., Christensen, M., Davis, P., Falin, L. J., Grabmueller, C., Hughes, D. S. T., Humphrey, J., Kerhornou, A., Khobova, J., Langridge, N., McDowall, M. D., Maheswari, U., Maslen, G., Nuhn, M., Ong, C. K., Paulini, M., Pedro, H., Toneva, I., Tuli, M. A., Walts, B., Williams, G., Wilson, D., Youens-Clark, K., Monaco, M. K., Stein, J., Wei, X., Ware, D., Bolser, D. M., Howe, K. L., Kulesha, E., Lawson, D., and Staines, D. M. 2014. Ensembl genomes 2013: Scaling up access to genome-wide data. Nucleic Acids Res. 42:D546-D552.

Line, R. F. 2002. Stripe rust of wheat and barley in North America: A retrospective historical review. Annu. Rev. Phytopathol. 40:75-118.

Line, R. F., and Qayoum, A. 1992. Virulence, aggressiveness, evolution, and distribution of races of Puccinia striiformis (the cause of stripe rust of wheat) in North America, 1968-87. U. S. Dep. Agric. Agric. Res. Serv. Tech. Bull. No. 1788.

Low, P. S., and Merida, J. R. 1996. The oxidative burst in plant defense: Function and signal transduction. Physiol. Plant. 96:533-542.

Marshall, D., and Sutton, R. L. 1995. Epidemiology of stripe rust, virulence of Puccinia striiformis f. sp. hordei and yield loss in barley. Plant Dis. 79: 732-737.

McGrann, G. R. D., Stavrinides, A., Russell, J., Corbitt, M. M., Booth, A., Chartrain, L., Thimas, W. T. B., and Brown, J. K. M. 2014. A trade off between $m l o$ resistance to powdery mildew and increased susceptibility of barley to a newly important disease, Ramularia leaf spot. J. Exp. Bot. 65: 1025-1037.

McNeal, F. H., Konzak, C. F., Smith, E. P., Tate, W. S., and Russell, T. S. 1971: Pages 34-121 in: A Uniform System for Recording and Processing Cereal Research Data. United States Department of Agriculture-Agricultural Research Service, Washington, DC.

Mhamdi, A., Queval, G., Chaouch, S., Vanderauwera, S., Van Breusegem, F., and Noctor, G. 2010. Catalase function in plants: A focus on Arabidopsis mutants as stress-mimic models. J. Exp. Bot. 61:4197-4220.

Morton, N. E. 1998. Levels in complex inheritance. Am. J. Hum. Genet. 62: 690-697.

Muir, C. E., and Nilan, R. A. 1973. Registration of Steptoe barley. Crop Sci. 13:770.

Muñoz-Amatriaín, M., Cuesta-Marcos, A., Endelman, J. B., Comadran, J., Bonman, J. M., Bockelman, H. E., Chao, S., Russell, J., Waugh, R., Hayes, P. M., and Muehlbauer, G. J. 2014. The USDA barley core collection: Genetic diversity, population structure, and potential for genome-wide association studies. PLoS One 9:e94688.

Navarro, F. M., and Zamora, M. D. 1990. Control quimico de la roya lineal amarilla de la cebada en la Mesa Central. SARH. INIFAP-CIFAEM, Mexico City, Mexico.

Niks, R. E., Alemu, S. K., Marcel, T. C., and van Heyzen, S. 2015. Mapping genes in barley for resistance to Puccinia coronata from couch grass and to P. striiformis from brome, wheat and barley. Euphytica 206:487-499.

Obert, D. E., Evans, C. P., Wesenberg, D. M., Windes, J. M., Erickson, C. A., Jackson, E. W., Chen, X. M., and Whitmore, J. C. 2008. Registration of 'Lenetah' spring barley. J. Plant Reg. 2:85-87.

Peterhänsel, C., and Lahaye, T. 2005. Be fruitful and multiply: Gene amplification inducing pathogen resistance. Trends Plant Sci. 10:257-260.

Richardson, K. L., Vales, M. I., Kling, J. G., Mundt, C. C., and Hayes, P. M. 2006. Pyramiding and dissecting disease resistance QTL to barley stripe rust. Theor. Appl. Genet. 113:485-495.

Stotz, H. U., Thompson, J. G., and Wang, Y. 2009. Plant defensins. Defense, development and application. Plant Signaling Behav. 4:1010-1012.

Suzuki, N., Miller, G., Morales, J., Shulaev, V., Torres, M. A., and Mittler, R. 2011. Respiratory burst oxidases: The engines of ROS signaling. Curr. Opin. Plant Biol. 14:691-699.

Toojinda, T., Baird, E., Booth, A., Broers, L., Hayes, P., Powell, W., Thomas, W., Vivar, H., and Young, G. 1998. Introgression of quantitative trait loci (QTLs) determining stripe rust resistance in barley: An example of markerassisted line development. Theor. Appl. Genet. 96:123-131.

Toojinda, T., Broers, L. H., Chen, X. M., Hayes, P. M., Kleinhofs, A., Korte, J., Kudrna, D., Leung, H., Line, R. F., Powell, W., Ramsay, L., Vivar, H., and Waugh, R. 2000. Mapping quantitative and qualitative disease resistance genes in a doubled haploid population of barley (Hordeum vulgare). Theor. Appl. Genet. 101:580-589.

Vales, M. I., Schon, C. C., Capettini, F., Chen, X. M., Corey, A. E., Mather, D. E., Mundt, C. C., Richardson, K. L., Sandoval-Islas, J. S., Utz, H. F., and Hayes, P. M. 2005. Effect of population size on the estimation of QTL: A test using resistance to barley stripe rust. Theor. Appl. Genet. 111:1260-1270.

Wesenberg, D. M., Burrup, D. E., Brown, W. M., Jr., Velasco, V. R., Hill, J. P., Whitmore, J. C., Karow, R. S., Hayes, P. M., Ullrich, S. E., and Liu, C. T. 2001. Registration of Bancroft barley. Crop Sci. 41:265.

Yan, G. P., and Chen, X. M. 2006. Molecular mapping of a recessive gene for resistance to stripe rust in barley. Theor. Appl. Genet. 113:529-537. 